\title{
Éduquer : une double responsabilité ?
}

L'exemple de la Hesse

Recognising responsibilities on th sides - Parent participation in Hesse, Germany Educar : ¿una doble responsabilidad? El ejemplo de Hesse

\section{Dorothea Schlegel-Hentrich}

Traducteur : Sandrine Lamer

\section{(2) OpenEdition}

\section{Journals}

Édition électronique

URL : https://journals.openedition.org/ries/1962

DOI : $10.4000 /$ ries. 1962

ISSN : 2261-4265

Éditeur

France Education international

Édition imprimée

Date de publication : 1 décembre 2002

Pagination : 109-116

ISBN : 978-2854-2-0555-8

ISSN : 1254-4590

\section{Référence électronique}

Dorothea Schlegel-Hentrich, «Éduquer : une double responsabilité ? », Revue internationale d'éducation de Sèvres [En ligne], 31 | décembre 2002, mis en ligne le 25 novembre 2011, consulté le 09 juillet 2021. URL : http://journals.openedition.org/ries/1962 ; DOI : https://doi.org/10.4000/ries.1962 


\section{Éduquer : une double responsabilité ?*}

\section{L'exemple de la Hesse}

\section{Dorothea Schlegel-Hentrich}

En Allemagne, la responsabilité du système éducatif revient à l'État et aux Länder. Les écoles privées restent une exception et le coût élevé des frais de scolarité ne permet qu'à un groupe restreint de la société d'y accéder.

La responsabilité politique et donc les contenus des enseignements incombent, eux, au ministère de l'Éducation et des Cultes (Kultusministerium) de chaque Land, du fait de la structure fédérale allemande. Les choix politiques du gouvernement de chaque Land, dont dépendent les décisions prises pour la vie scolaire, relèvent du parti au pouvoir. Les deux grands partis allemands dominant le paysage politique, les sociaux démocrates (SPD) et les chrétiens démocrates (CDU), représentent les deux «camps principaux» du positionnement pour l'école : la SPD s'engage à offrir «les mêmes chances d'éducation à tous les enfants"; la CDU donne la priorité à la sélection et à l'éducation élitiste. Les mêmes divergences se rencontrent au niveau des objectifs pour le développement du système scolaire de chaque Land. Comme chaque période de législature dure quatre ans, des changements fondamentaux en ce qui concerne l'institution scolaire peuvent, dans le pire des cas, intervenir tous les quatre ans, avec toutes les conséquences que cela entraîne. Pour les parents, cela signifie que l'offre du système éducatif tout comme la qualité des formations proposées dépendent du Land dans lequel ils vivent.

Quel que soit le Land, la majorité des enfants sont scolarisés dans les établissements publics. Pour les parents, deux points sont importants : une hiérarchie rigoureuse est à la base de l'institution scolaire et les cours n'occupent que les demi-journées ${ }^{1}$.

\section{LES DROITS DE CO-DÉCISION DES PARENTS}

Il existe dans tous les Länder des réglementations fixées par la loi concernant les droits de co-décision des parents, leur principe étant que parents et école ont ensemble un devoir éducatif d'égale valeur. Ce droit des parents constitue la base de cette "coopération ingénieuse sur une base de réciprocité», indispensable, entre parents et école.

\footnotetext{
* Cet article a été traduit par Sandrine Lamer.

1. NDLR : principalement dans les anciens Länder.
} 
La Hesse est le seul Land dans lequel une valeur constitutionnelle ait été octroyée à cette coopération et dans lequel les tuteurs légaux aient un droit de co-décision sur l'organisation de l'éducation. L'application de ces droits repose sur une structure particulière de parents délégués.

Au départ, il y a l'ensemble des parents d'élèves d'une classe, parmi lesquels tous les deux ans deux délégués sont élus. Leur devoir est de s'occuper des intérêts des enfants et des parents de cette classe. Parmi leurs responsabilités, il leur revient d'organiser au moins deux réunions de parents d'élèves par an. Ils doivent faire le lien entre les parents d'élèves et les enseignants de leur classe. Ils sont membres du «conseil général des parents d'élèves»(Gesamtelternbeirat), c'est-à-dire une commission dans laquelle sont représentés tous les conseils de parents par classe. Le conseil général des parents d'élèves élit en son sein un conseil d'administration avec un président du conseil parents/école. Ce conseil d'administration, et en particulier le président, doivent présenter et représenter les intérêts des conseils de parents d'élèves vis-à-vis de la direction de l'établissement. Le conseil des parents d'élèves doit donc faire part au conseil général des difficultés qu'il n'aura pas pu résoudre au sein de la classe.

Le conseil général des parents d'élèves est l'une des commissions de co-décision de l'institution scolaire. Par ailleurs, la «conférence générale» (Gesamtkonferenz) rassemble tous les enseignants et la «délégation d'élèves» (Schüler110 vertretung) est organisée dans sa structure comme celle des parents. Le conseil général des parents d'élèves dispose de plusieurs droits de co-décision : il peut avoir à donner son accord, à être à l'écoute des différents acteurs, à faire des propositions, à informer et même à contester une décision. Ces droits sont inscrits dans la loi scolaire qui dépend des représentations idéologiques du gouvernement de chaque Land en ce qui concerne l'institution scolaire et la famille et qui, selon les changements de gouvernement, évolue régulièrement entre restriction et conservatisme ou bien progressisme et innovation. L'actuelle majorité conservatrice en Hesse a par exemple, dès son arrivée au pouvoir, réduit considérablement les droits des parents d'élèves introduits auparavant par les progressistes puisque, d'après leur conception de l'éducation, il revient aux parents de s'acquitter de ce travail à la maison et de «livrer» à l'école des enfants «bien» éduqués. Ainsi, le rôle de l'école peut se limiter à la transmission du savoir.

Les trois membres de la communauté scolaire (Mitglieder der Schulgemeinde), enseignants, élèves et parents, élisent un représentant de leur groupe présent dans la «conférence scolaire»(Schulkonferenz) qui, en tant que commission supérieure sous la présidence du chef d'établissement, tient conseil et prend les décisions. Les obligations et les droits de cette commission sont également soumis à de fortes fluctuations selon la majorité du gouvernement, sa perception des domaines de compétences attribuables aux élèves et aux parents au sein des établissements scolaires étant extrêmement variable. Les conservateurs sont d'avis qu'une régulation par le haut assure une plus grande qualité de vie 
scolaire, les progressistes partent du principe que la dé-hiérarchisation liée à une plus grande autonomie de chaque école constitue le seul moyen de moderniser l'institution scolaire et de la faire évoluer dans l'intérêt de tous les enfants. Élèves et enseignants restent toujours les victimes de ce va-et-vient idéologique, bien que les différents points de vue prétendent aller dans le sens de leur intérêt.

Outre les instances représentatives propres à chaque établissement, il existe d'autres commissions de représentants des parents d'élèves.

Le niveau suivant correspond aux grandes villes ou encore au territoire intercommunal, pour lesquels est élu au sein de tous les conseils généraux de parents d'élèves concernés un "conseil des parents d'élèves par ville» (Stadtelternbeirat) ou un «conseil des parents d'élèves par territoire intercommunal» (Landeselternbeirat). Ces commissions prennent en considération toutes les requêtes des établissements scolaires vis-à-vis des circonscriptions académiques (Schulämter), elles-mêmes responsables du financement de l'équipement et de la construction des établissements scolaires, et vis-à-vis des circonscriptions dites académiques d'État, chargées d'assurer et de contrôler les contenus et les méthodes d'enseignement des établissements scolaires, en particulier en ce qui concerne la répartition des enseignants.

C'est également parmi les conseils généraux des parents d'élèves de chaque école que le «conseil fédéral des parents d'élèves» (Landeselternbeirat) est élu. Il est le partenaire du ministère de l'Éducation et de la Culture (Kultusministerium), c'est-à-dire l'instance qui définit les lignes de conduite pour tous les établissements scolaires en matière de programmes, de structures scolaires, de recrutement des enseignants, de formation continue, de droits parentaux, d'examens de fin d'études, de modalités d'évaluation (notes, bulletins scolaires), d'effectifs d'élèves par classe et de possibilités de développement des établissements. En Hesse, le conseil des parents d'élèves par territoire intercommunal est, au contraire d'autres Länder, une commission indépendante disposant de droits de cogestion, en particulier lorsqu'il s'agit d'introduire de nouvelles réglementations et de nouveaux décrets juridiques. Le conseil intervient pour les décisions sur les modalités d'application des lois.

Le travail de toutes ces commissions ne consiste pas seulement à donner un avis sur ce qui vient d'autres commissions ou de la direction des établissements scolaires. Elles doivent également prendre des initiatives, proposer des thèmes de réflexion et donner des impulsions. Leur efficacité dépend inévitablement de leurs acteurs, car plus ceux-ci s'engagent de manière offensive dans leur fonction, plus ils ont d'influence et plus ils sont efficaces.

\section{LA PARTICIPATION AU-DELÀ DES RÉGLEMENTATIONS JURIDIQUES}

Pour les parents actifs, il existe en plus du travail dans les commissions d'autres possibilités de s'engager dans la vie scolaire. Ils peuvent aussi bien 
travailler sur des thèmes choisis qu'organiser des dons, des fêtes et diverses manifestations. Toutes les commissions de l'établissement scolaire peuvent par exemple constituer des comités qui se pencheront sur des questions spécifiques comme la prévention contre les drogues, la décoration de l'école ou l'apprentissage de la vie en société. Toute personne intéressée peut y participer. Les parents qui ont des compétences spécifiques du fait de leur formation ou de leur emploi, par exemple dans le domaine des nouvelles technologies, du commerce ou des langues, peuvent soit proposer d'autres thèmes, avec l'autorisation du chef d'établissement, soit intervenir dans les cours.

Les parents peuvent fonder leur propre association de parents d'élèves qui collecte de l'argent grâce aux cotisations et aux dons. Ces sommes permettent d'investir dans du matériel pour l'école qui ne peut pas être financé par des fonds publics : des appareils pour les activités ludiques et sportives ou encore des appareils pour les sciences naturelles. Des exposés spécialisés peuvent être organisés sur des thèmes intéressant parents et élèves comme «la puberté » ou «le harcèlement en classe».

Les parents peuvent offrir leur participation pour améliorer le climat dans l'enceinte de l'établissement en s'engageant dans l'organisation de manifestations en tous genres qui permettent à la communauté scolaire de se rencontrer en dehors des cours. C'est dans ce cadre qu'on trouve les fêtes de l'école, les bals, les brocantes, les manifestations à bénéfices ou les concerts.

\section{LE PROJET D’ÉTABLISSEMENT}

En Hesse, toutes les écoles jusqu'à cette dernière rentrée scolaire devaient présenter un projet d'établissement. Son objectif est de définir un profil propre à chaque établissement scolaire lui permettant de déterminer ses spécificités et de se fixer de nouveaux objectifs. Ce projet doit être conçu en concertation avec les enseignants, les parents et les élèves afin d'assurer un processus d'identification avec l'école et la réalisation des objectifs. Par ailleurs, le projet est évalué en interne et complété régulièrement sur cette base.

Les rapports des parents ayant participé à ces activités montrent que les institutions scolaires ont des modes de travail très variables car il était recommandé, mais non imposé, de faire intervenir les parents et les élèves dans le processus d'élaboration du projet. Dans de nombreuses écoles, pendant plusieurs années, le groupe d'enseignants chargé de s'en occuper présentait le projet à la commission des parents d'élèves juste avant la date limite de remise du dossier. De ce fait, la participation des parents était forcément limitée : ils n'avaient plus qu'à constater qu'on ne s'intéressait pas à leur position ni à leur opinion concernant leur école. D’autres écoles, qui ont pour principe de faire participer les parents, ont élaboré avec succès le projet de l'établissement en 
tenant compte de cet esprit. D'autres encore ont profité de cette occasion pour introduire sérieusement pour la première fois les parents dans la discussion.

La coopération des parents dans les commissions scolaires prévue par la législation est un acte bénévole. En revanche, aucune réglementation juridique ne prévoit de libérer les parents de leur lieu de travail pour qu'ils soient en mesure de mener à bien ces activités qui ne peuvent pas toujours se faire le soir. Le cercle des personnes susceptibles de proposer leurs services est donc inévitablement restreint car il n'est pas possible à chacun de quitter son lieu de travail pour prendre ses fonctions de parent d'élèves. De plus, le travail de délégué de parents d'élèves exige une qualification propre et un bon niveau d'information sur l'actualité. C'est particulièrement important en cas de collaboration difficile avec l'établissement scolaire. C'est pourquoi les associations de parents, les syndicats, les universités populaires, les églises et les institutions étatiques de formation continue pour les enseignants proposent aux parents des sessions d'information et du matériel spécifique.

\section{Partenariat en Éducation}

En Allemagne, le législateur part du principe que les institutions scolaires et les parents ont un «devoir éducatif commun». Celui-ci doit être mis en pratique au moyen du "partenariat en éducation». Il exprime ainsi l'idée d'une égalité des enseignants et des parents qui ont "pour objectif le développement de la seule personnalité de l'enfant» (citation du jugement final du tribunal constitutionnel de la République fédérale de 1972). Pourtant, l’institution scolaire persiste à croire que son rôle est avant tout celui de la transmission du savoir plus que celui de l'éducation des enfants. Elle limite donc son action à l'enseignement dont la méthode suppose que les enfants, généralement scolarisés à l'âge de six ans, sont, dès leur arrivée à l'école, capables de se concentrer, d'apprendre, de faire des efforts et surtout de maîtriser leur comportement social et leur attitude de travail en groupe.

Depuis quelque temps, de plus en plus d'écoles se plaignent que les enfants "ne sont plus éduqués». L'attitude des jeunes face au travail et leur comportement social sont à l'origine de ce constat. Les critiques sont les suivantes : capacité de concentration et efforts insuffisants, devoirs régulièrement non faits, absence de discipline, incapacité à gérer les conflits, grand potentiel de violence, incivilité et manque absolu d'aptitude à comprendre les autres, égoïsme massif, absentéisme. Du côté des enseignants, tout cela est perçu comme la conséquence d'une éducation lacunaire dans la famille à laquelle ils renvoient donc aussitôt la charge d' "élever enfin correctement» leurs enfants.

Il existe indéniablement un affaiblissement de la rigueur éducative dans un nombre croissant de familles en Allemagne. On le remarque entre autres par le fait que, visiblement, les centres d'information et les services sociaux pour 
mineurs sont de plus en plus souvent appelés à résoudre des problèmes d'éducation au point de devoir proposer des "aides à l'éducation", c'est-à-dire une prise en charge des enfants dans des foyers.

C'est le manque d'assurance des parents qui est à la base de ces problèmes et qui engendre leur sentiment d'impuissance et les pousse à baisser les bras et à laisser leurs enfants livrés à eux-mêmes. Cette tendance touche maintenant toutes les couches de la société.

Deux éléments principaux sont à l'origine de cette situation. D’abord, il aurait fallu que, suite à l'éclatement des structures éducatives rigides et autoritaires, enfin réalisé à la fin des années soixante, s'établissent un schéma et des méthodes démocratiques pour l'éducation fondés sur le travail en partenariat. Cela ne s'est pas produit. Au contraire, les parents de l'actuelle génération de parents étaient eux-mêmes seuls à décider si leurs enfants pouvaient se développer librement ou s'ils devaient leur imposer des limites.

Ensuite, depuis les années quatre-vingt, cette évolution va de pair avec un changement radical de la société et de l'économie du fait de la globalisation. Sur ce même rythme, un glissement fondamental des valeurs s'est entre-temps introduit au cœur du domaine familial. Flexibilité, mobilité, précarité sont des mots clés qui jouent un grand rôle dans ce contexte car ils sont à l'opposé de ce dont les enfants ont besoin pour le bon développement de leur personnalité : 114 continuité, engagement, sécurité, long terme. Il semble que ce soit trop demander que d'exiger des seuls parents, de plus en plus souvent engagés tous deux dans la vie active, cette performance qui consiste à balancer entre deux pôles incompatibles pour que leurs enfants en ressortent «bons" pour l'école et ses modes actuels de fonctionnement.

Jusqu'à présent, en Allemagne, l'institution scolaire ne s'est pas mesurée à cette évolution : ni les méthodes, ni les contenus ne sont adaptés à la situation d'aujourd'hui. Aucune structure systématique de communication - qui, compte tenu des attentes dans le domaine éducatif, s'impose d'urgence n'a été établie avec les parents. On paie aujourd'hui les conséquences de cette négligence. Jusqu'à présent, les rencontres entre les enseignants et les parents avaient lieu en dehors du travail des commissions scolaires, et uniquement lors de l'apparition de difficultés de l'enfant ou avec l'enfant. Comment les parents pouvaient-ils se sentir les bienvenus?

Il s'agit maintenant de développer une réflexion qui tienne compte de deux nouveaux paramètres : le comportement des parents en matière d'éducation a changé et on ne peut plus motiver les enfants d'aujourd'hui dans leurs apprentissages avec des méthodes et des contenus d'enseignement d'hier. Il faut tirer la leçon du désintérêt croissant des élèves pour l'école et de leur recours à l'absentéisme sous toutes ses formes.

Le principe de l'«avance sur le savoir» dont jouissaient les anciennes générations sur les plus jeunes n'est plus valable. L'institution scolaire doit 
aujourd'hui tenir compte de cette évolution. L'extrême rapidité du développement des technologies fait que les élèves en savent entre-temps souvent plus que leurs enseignants et leurs parents.

Il devient évident, à ce stade au moins, que l'institution scolaire doit abandonner sa conception hiérarchique et s'engager dans un véritable partenariat avec les parents. L'exemple du Land de Brême où l'administration scolaire pense à supprimer les allocations familiales si les parents ne parviennent pas à empêcher leur enfant de manquer les cours montre à quel point cela est difficile. On a là-bas, manifestement, abandonné l'idée de proposer une coopération à ces parents.

En Hesse, le ministère de l'Éducation et des Cultes conseille aux établissements scolaires de conclure un «contrat d'éducation». Pour ce faire, les enseignants doivent rédiger avec l'élève concerné et ses parents une "convention» concernant son attitude à venir et se mettre d'accord sur les sanctions à prendre en cas de non-respect de l'accord. Le grand danger de cette action est, en revanche, qu'il s'agit d'un «contrat» très unilatéral. Les enseignants ne sont en aucune manière tenus de le faire appliquer. On ne peut donc pas parler de véritable partenariat en éducation.

\section{DEMI-JOURNÉE, JOURNÉE COMPLÈTE?}

Lorsque les cours sont répartis sur une demi-journée en Allemagne, plusieurs matières sont enseignées sur des plages de cinq à six fois quarantecinq minutes, avec des pauses courtes et une récréation. Il ne reste ni espace ni temps pour la vie de communauté entre les élèves ou pour introduire des méthodes d'enseignement autres que le cours magistral. Pourtant, l'école constitue le seul environnement social de la plupart des enfants, et ce à cause des conditions dans lesquelles ils grandissent, qui sont totalement différentes de celles qui existaient autrefois : logement qui ne permet plus de jouer dehors, familles éclatées, familles monoparentales, familles dont les deux parents travaillent, absence de camarades du même âge "au coin de la rue » parce qu'il y a de moins en moins d'enfants, éloignement géographique des autres branches de la famille. C'est à l'école que les enfants rencontrent des jeunes de leur âge et ceux qu'ils voudraient bien ne pas voir qu'en classe. Mais lorsque l'école ne dure qu'une demi-journée, aucun espace n'est prévu pour cela. Au contraire, les enfants sont renvoyés chez eux avec leurs devoirs pour lesquels ils auraient besoin de l'aide de leurs parents. Ainsi, les enfants qui reçoivent cette aide de leurs parents, aussi bien en termes de temps passé que de contenus, ont plus de chances d'obtenir de bons diplômes que ceux qui ne reçoivent pas ce soutien. C'est ce que montrent clairement les résultats de l'étude PISA pour l'Allemagne. Les cours sur une demi-journée obligent les mères à rester au foyer, si tant est 
qu'elles s'intéressent à la réussite scolaire de leur enfant et qu'elles peuvent et veulent se permettre de ne pas être dans la vie active, afin de pouvoir lui préparer un repas le midi avant de lui donner des cours de soutien en reprenant et approfondissant les enseignements de la matinée.

En Allemagne, le débat sur l'introduction de la journée scolaire à plein temps est actuellement lancé et la discussion agitée. Il ne s'agit pas de pratiquer à l'école le même type de cours l'après-midi que le matin, mais de trouver un modèle qui permettrait de laisser de l'espace et du temps à l'enseignement par projets, ce qui rendrait possibles l'apprentissage social et des activités communes en lien avec les penchants et les intérêts particuliers des élèves. Une école de cette nature est fondée sur l'ouverture à l'environnement local et à la coopération avec les associations, les entreprises et les institutions. Tout cela n'est possible que sur la base d'un engagement de collaboration avec les parents.

Le développement du projet serait à charge individuelle de l'école qui est plus à même d'évaluer ses besoins spécifiques et qui connaît mieux son environnement.

Il faudra encore beaucoup de persuasion pour mettre en place une école dont les cours se répartissent de la sorte sur la journée, car les parents ne sont pas tous prêts à laisser leurs enfants toute la journée «à quelqu'un d'autre » et beaucoup d'enseignants n'y voient pas un allègement mais au contraire un surcroît de leur charge de travail. Cependant, quelques établissements scolaires travaillent déjà dans ce sens et les expériences semblent très positives.

Il est vraisemblable qu'en Allemagne, au cours des années à venir, de plus en plus d'écoles proposeront des cours pendant toute la journée. 